\title{
Recent Advances on Tunable Vortex Beam Devices for Biomedical Applications
}

\author{
Yijie Shen ${ }^{1,2}$, Xilin Yang ${ }^{3}$, Ruoyang $\mathrm{Qi}^{4}$, Zhensong Wan ${ }^{1,2}$, Xing Fu ${ }^{1,2}$ and Mali Gong*1,2,5 \\ ${ }^{1}$ Department of Precision Instruments, Tsinghua University, China \\ ${ }^{2}$ Key Laboratory of Photonic Control Technology (Ministry of Education), Tsinghua University, China \\ ${ }^{3}$ The School of Optics and Electronics, Beijing Institute of Technology, China \\ ${ }^{4}$ Department of Physics, Tsinghua University, China \\ ${ }^{5}$ Department of Mechanical Engineering, Tsinghua University, China
}

Received: 阱: September 21, 2018; Published: 制 September 28, 2018

*Corresponding author: Mali Gong, State Key Laboratory of Precision Measurement Technology and Instruments, Department of Precision Instruments, Key Laboratory of Photonic Control Technology (Ministry of Education), Tsinghua University, Beijing 100084, China

Abstract

The optical vortex beams carrying orbital angular momentum (OAM) recently have unveiled great potential to be widely applied in advanced biomedicine, e.g. manipulating and assembling DNA, detecting biomolecule, organic illumination, single-cell nanosurgery, etc. However, it is an everlasting challenge to produce the proper vortices devices for corresponding applications. To this end, the tunable properties of OAM beams need to be produced. The spectrum-tunable property is related to the tuning of the response of target molecules. The OAM-tunable property can control the number and distribution of target particles. Moreover, the polarization-tunable property can be used to reduce photodamage. Hereinafter, we give a prompt review of the recent advances on tunable vortex beam devices for biomedical applications.

Abbreviations: OAM: Orbital Angular Momentum; AIFG: Acoustically-Induced Fiber Grating; MEMS: Micro-Electro-Mechanical-System; HLG: Hermite-Laguerre-Gaussian; HIG: Helical-Ince-Gaussian; AMC: Astigmatic Laser Mode Converter; PWE: Paraxial Wave Equation; LCD: Liquid-Crystal Display; SLM: Spatial Light Modulator

\section{Mini Review}

Optical vortex beams are at the heart of a number of novel research directions, which are the light beams with a helical wavefront carrying orbital angular momentum (OAM), giving rise to a phase singularity at the center of light field [1-3]. In 2004, X Zhuang [4] unveiled that a DNA molecule can be manipulated by a vortex beam which plays a role of optical tweezer. Moreover, she discovered that the position, rotation, even condensation of the target DNA or other biomolecule can be flexibly controlled so as to do biomolecules assembling engineering via tuning the corresponding vortex beam [5-8]. With more researches on the interaction between matters and optical OAM hatched in recent years, vortex beams were gradually developing more and more powerful advanced applications in biomedicine [9]. Utilizing the coupling between the chirality of molecule and the chirality of OAM of vortex beams [10-12], one can detect, select and sort the target molecules with special chirality or enantiomorphism, offering an alternative to existing bio-sensing technologies $[13,14]$. With the development of the tunable vortex beams in nanometersized subcellular structures [15-17] and the optimization of the polarization-tunable property [18-20], the optical tweezers developed the functionality of transporting subcellular organelles and exerting less photodamage on the trapped particle, which has been used in advanced single-cell nanosurgery [21].

In 2006, F Tamburini et al. [22] unveiled that the Rayleigh criterion limit (diffraction limit) can be overcome with optical vortices in imaging process, which extend the novel super-resolution imaging technology using vortex beams. Then, researchers reported that the resolution in this super-resolution imaging can be further improved by using optical vortex lattices i.e. the vortex beam with multiple singularities and singularity arrays [23]. Moreover, through tuning the polarization structure of the vortex beam, the resolutions of the superresolution imaging technology can be close to $100 \mathrm{~nm}$, which is capable of clearly observing biological cells $[24,25]$. Therefore, the development of large-range tunable vortex beams can largely improve the super-resolution imaging for the application of biomedical photonics. In this year, a breakthrough in vortices generation is that the vortex beam can be generated in organic material [26], which has great potential to develop 
novel organic illumination technologies and further be used in microimaging subcellular dynamics in multicellular organisms [27] in the future.

According to the above review, the development of tunable vortex beams largely promoted the related biomedical applications. In contrast to the conventional fundamental mode beam, the vortex beams have spatial structures with structured OAM and polarization properties. Thus, besides the conventional wavelength-tunable property, its tunable properties also include OAM-tunable and polarization-tunable properties. All these three kinds of tunable properties provided distinct directions for improving the related biomedical applications. Therefore, it is meaningful to investigate the vortex beams devices with more powerful tunable properties. For the wavelength- and OAM-tunable vortex beams devices, recent advances can be divided into two categories, single-singularity topological charge tuning and multiple singularities distribution tuning. The former one will be discussed first. Although the technologies for wavelength-tunable and OAM-tunable lasers are existed for a long time, yet the generation of wavelength and OAM simultaneously tuning lasers is a cutting-edge topic.

In 2016, W Zhang et al. [28] developed a method for the optical vortex generation with wavelength tunability via an acousticallyinduced fiber grating (AIFG) driven by a radio frequency source. By tuning the frequency of RF driving signal, topological charge of the generated optical vortex can be converted to $0 \sim \pm 1 \hbar$ within the wavelength range $1540 \mathrm{~nm}-1560 \mathrm{~nm}$. However, the OAM tuning range is limited to $0 \sim \pm 1 \hbar$, leading to scant applications. In 2017 , V Lyubopytov et. al. [29] expand the OAM tunable range to $0 \sim 3 \hbar$ by using a new on-chip micro-component - tunable Micro-ElectroMechanical-System (MEMS)-based Fabry-Perot filter integrated with a spiral phase plate. In the same year, Q Liu et al. [30] reported a design of a wavelength- and OAM-tunable vortex beam in Er:YAG laser where the tunable parameters are $8.4 \mathrm{~nm}$ range of wavelength and $0 \sim \pm 2 \hbar$ OAM. In early 2018, our group [31,32] presented a dual-off-axis pumping scheme in Yb:CaGdAlO4 (Yb:CALGO) lasers with external mode converter to generate wavelength- and OAMtunable vortex beams, with wavelength-tunable range across 10 $\mathrm{nm}$ and a large OAM-tunable range of $0 \sim \pm 15 \hbar$, which for the first time breaks ten-level OAM range in wavelength- and OAM-tunable vortex beams to the best of our knowledge. The whole system is free of extra tuning devices and the light source is simply structured and easy to implement on a relatively low cost.

Later in 2018, S Wang et al. [33] also adopted the similar method to generate wavelength- and OAM-tunable vortex beams. Moreover they employed a z-type cavity design to realize very low threshold and a thin film polarizer to precisely control the center wavelength. The final tunable range reached $0 \sim \pm 14 \hbar$ for OAM and $14.5 \mathrm{~nm}$ for wavelength-tunable width. Around the same time, N Zhou et al. [34] designed and implemented a fiber-free space hybrid coupling laser device with wavelength and OAM tunable properties which solved the problem that large OAM is difficult to be tuned in fiber lasers. The OAM tunable range is $0 \sim \pm 10$ h while the wavelength can be tuned to cover the entire C-band range of $1530-1565 \mathrm{~nm}$. This is the first demonstration of ten-level OAM tunable lasers using fiber systems.

Compared with single-singularity topological charge tuning, multiple singularities topological charge tuning is much more sophisticated as it not only involves topological charge tuning but also involves singularities distribution manipulation, which plays a crucial role in optical tweezers and multi-particle manipulation. Although singularity splitting was found in mode converter several years after OAM was proposed, how to generate vortex beams with multiple singularities remains a hot research topic. To the best of our knowledge, absolute arbitrary control is not yet actualized, but various types of such vortex beams, their theory and generation methods have been reported including Hermite-Laguerre-Gaussian (HLG) beams [35-37], Helical-Ince-Gaussian (HIG) beams [38,39], fractional vortex beams [40-42], SU(2) wave-packet [43-45], and polygonal vortex beams $[46,47]$. Using an astigmatic laser mode converter(AMC) [48] was the earliest way to produce vortex beams. When the phase matching condition in the AMC is not strictly satisfied, singularly splitting phenomenon will appear in the output light field, forming HLG modes [35-37].

HG and LG beams are the solutions of paraxial wave equation (PWE) separable in Cartesian and circular cylindrical coordinates, respectively, while IG beams, proposed in 2004 [38], are the solutions of elliptical or hyperbolic coordinates, constituting the continuous transition modes between HG and LG beams. Soon later HIG modes were theoretically constructed exhibiting helical phase features as with LG beams [39] and then practically generated by using complex amplitude and phase masks encoded onto a liquidcrystal display (LCD) [49]. As a kind of multi-singularity beams, HIG beams were further successfully applied in optical trapping [50]. In 2004, vortex beams with fractional OAM were proposed [40,41], which has been a controversial topic. To physically demonstrate fractional OAM, topological charge is not comprehensive and various methods including OAM spectrum measurement [51,52], intrinsic and external OAM[53]. Similar to the famous mathematical paradox, the propagation of light through fractional vortex plates are descripted as fractional vortex Hilbert's Hotel[54].

However, G. Tkachenko et al. questioned the existence of perfect fractional vortex beams [55] and claimed that one cannot create perfect fractional vortex beams because of its phase and intensity disturbance introduced by fractional indexes which cannot be smoothed out. Anyhow, fractional OAM gave a peculiar family of multi-singularities vortex beams. As another special kind of multisingularities vortex beams, SU(2) geometry modes are introduced by Y.F. Chen and attract much attention with its special features such as localized multi-path periodic ray trajectories, unusual power peaks, and as mentioned above, large fractional OAMs [43-45]. Recently, our group also exploited a new kind of multi-singularity beams: polygonal vortex beams $[46,47]$, which is generated by a quasi-frequency-degenerate laser resonator with astigmatic transformation. These various kinds of multi-singularity beams represents the development of complex OAM distribution tuning technologies. For the polarization-tunable vortex beams devices, 
the main tuning technologies focus on the control of polarization distribution.

The vortex beams can be classified into vector and scalar vortex beams [56,57]. The distribution of polarization is homogenous and inhomogeneous for a scalar vortex beams and a vector vortex beams, respectively. Vector vortex beams are paraxial solutions of the vector Helmholtz equation [58]. Special families of scalar vortex beams and vector vortex beams are illustrated by a point of high-order poincaré sphere [59]. In the past several years, Light beams with an inhomogeneous state of polarization have attracted much attention. Investigations and interest focused on the vector beams with radial and azimuthal polarizations. Such beams can be generated by an intracavity $[60,61]$ or an external approach $[62-$ 67]. An example of intracavity method is a work of VG. Niziev's group [60]. They obtained the controlled vector vortex beams by using an intracavity Sagnac interferometer with a thin wire line across the center of the cavity was used to control the laser mode.

In the last decade, there are many ways to generate a vector vortex beam with more kinds of polarization structures outside the cavity. In 2016, D Naidoo et. al [68] designed a versatile method to generate arbitrary beams of high-order Poincaré sphere through intracavity q-plate controlling. For the external methods, vector vortex beams have been experimentally obtained by Q-plate or the combination of q-plate and spiral phase plate [62], radial/azimuth analyzer and radial/azimuth polarization $[63,64]$, and the optically active plate [64]. However, these plates lack of tunable flexibility. With the development of programmable devices, spatial light modulator (SLM) is increasingly being utilized to generate vector vortex beams [65-67]. In 2017, a novel interferometric approach to generate arbitrary vector vortex beams on the high-order Poincaré sphere has been proposed by Shizhen Chen et al. [67]. In this year, more and more kinds of special structured polarized beams were successively reported with the control of the modulation of SLM [69-72].

To date, the vortex source devices with various tunable properties are successively reported, however, it is still a challenge to tailor a versatile method for all the wavelength-, OAM-, and polarization-tunable properties. Therefore, in the future, the appearance of novel tunable vortex source devices with more superior property and flexibility will have great potential to largely promote and develop more advanced biomedical technologies and applications.

\section{References}

1. Coullet P, Gil L, Rocca F (1989) Optical vortices. Opt Commun 73(5): 403408.

2. Allen L, Beijersbergen MW, Spreeuw RJC, Woerdman JP (1992) Orbital angular momentum of light and the transformation of LaguerreGaussiean laser mode. Phys Rev A 45(11): 8185.

3. Yao AM, Padgett MJ (2011) Orbital angular momentum: origins, behavior and applications. Adv Opt Photonics 3(2): 161.

4. Zhuang X (2004) Molecular biology. Unraveling DNA condensation with optical tweezers. Science 305: 188-190.
5. Padgett M, Bowman R (2011) Tweezers with a twist. Nat Photonics 5(6): 343-348.

6. Dholakia K, Čižmár T (2011) Shaping the future of manipulation. Nat Photonics 5(6): 335-342.

7. Grier DG (2003) A revolution in optical manipulation. Nature 424(6950): 810-816.

8. Paterson L, MacDonald MP, Arlt J, Sibbett W, Bryant PE, et al. (2001) Controlled rotation of optically trapped microscopic particles. Science 292: 912-914.

9. Zhou R, Wang H, Zhu D, Hu X (2017) New advances in the applacation of optical Tweezers in biology. Acta Laser Biol Sin 26(4): 289-293.

10. Forbes KA, Andrews DL (2018) Optical orbital angular momentum: twisted light and chirality. Opt Lett 43(3): 435.

11. Wang SB, Chan CT (2014) Lateral optical force on chiral particles near a surface. Nat Commun 5: 1-8.

12. Andrews DL, Romero LCD, Babiker M (2004) On optical vortex interactions with chiral matter. Opt Commun 237(1-3): 133-139.

13. Brullot W, Vanbel MK, Swusten T, Verbiest $T$ (2016) Resolving enantiomers using the optical angular momentum of twisted light. Sci Adv 2(3): 1-6.

14. Zhao Y, Askarpour AN, Sun L, Shi J, Li X, et al. (2017) Chirality detection of enantiomers using twisted optical metamaterials. Nat Commun 8: 6-13.

15. Ostrovsky E, Cohen K, Tsesses S, Gjonaj B, Bartal G (2018) Nanoscale control over optical singularities. Optica 5(3): 283-288.

16. Toyoda K, Takahashi F, Takizawa S, Tokizane Y, Miyamoto K (2013) Transfer of light helicity to nanostructures. Phys Rev Lett 110(14): 1-5.

17. Cai X, Wang J, Strain MJ, Johnson Morris B, Zhu J, et al. (2012) Integrated Compact Optical Vortex Beam Emitters. Science 338(6105): 363-366.

18. Zhan Q (2009) Cylindrical vector beams: from mathematical concepts to applications. Adv Opt Photonics 1(1): 1.

19. Otsuka K, Chu SC (2013) Microchip solid-state cylindrical vector lasers with orthogonally polarized dual laser-diode end pumping. Opt Lett 38(9): 1434.

20. Moh KJ, Yuan XC, Bu J, Burge RE, Gao BZ (2007) Generating radial or azimuthal polarization by axial sampling of circularly polarized vortex beams. Appl Opt 46: 7544-7551.

21. Jeffries GDM, Edgar JS, Yiqiong Z, Shelby JP, Christine F (2007) Using polarization-shaped optical vortex traps for single-cell nanosurgery. Nano Lett 7(2): 415-420.

22. Tamburini F, Anzolin G, Umbriaco G, Bianchini A, Barbieri C (2006) Overcoming the Rayleigh criterion limit with optical vortices. Phys Rev Lett 97(16): 1-4.

23. Xie X, Chen Y, Yang K, Zhou J (2014) Harnessing the point-spread function for high-resolution far-field optical microscopy. Phys Rev Lett 113(26): 1-5.

24. Kozawa Y, Matsunaga D, Sato S (2018) Superresolution imaging via superoscillation focusing of a radially polarized beam. Optica 5(2): 86.

25. Zhang C, Min C, Du L, Yuan XC (2016) Perfect optical vortex enhanced surface plasmon excitation for plasmonic structured illumination microscopy imaging. Appl Phys Lett 108(20): 1-6.

26. Stellinga D, Pietrzyk ME, Glackin JME, Wang Y, Bansal AK, et al. (2018) An Organic Vortex Laser. ACS Nano 12(3): 2389-2394.

27. Liu TL, Upadhyayula S, Milkie DE, Singh V, Wang K (2018) Observing the cell in its native state: Imaging subcellular dynamics in multicellular organisms. Science 360(6386). 
28. Zhang W, Wei K, Huang L, Mao D, Jiang B (2016) Optical vortex generation with wavelength tunability based on an acoustically-induced fiber grating. Opt Express 24(17): 363-366.

29. Lyubopytov VS, Porfirev AP (2017) Simultaneous wavelength and orbital angular momentum demultiplexing using tunable MEMS-based Fabry-Perot filter. Opt Express 25(9): 9634-9646.

30. Liu Q, Zhao Y, Ding M, Yao W, Fan X (2017) Wavelength- and OAM-tunable vortex laser with a reflective volume Bragg grating. Opt Express 25(19): 23312-23319.

31. Shen Y, Meng Y, Fu X, Gong M (2018) Wavelength-tunable Hermite Gaussian modes and an orbital-angular-momentum-tunable vortex beam in a dual-off-axis pumped Yb: CALGO laser. Opt Lett 43(2): 43-46.

32. Shen Y, Gong M (2018) Generation of wavelength- and orbital-angularmomentum tunable vortex beam in Yb: CALGO laser. Conf Lasers \& Electro-Optics (CLEO) JTh2A p. 181.

33. Wang S, Zhang S, Li P, Hao M, Yang H, et al. (2018) Generation of wavelength- and OAM-tunable vortex beam at low threshold. Opt Express 26(14): 213-214.

34. Zhou N, Liu J, Wang J (2018) Reconfigurable and tunable twisted light laser. Sci Rep 8(1): 1-10.

35. Alieva T (2004) Mode mapping in paraxial lossless optics. Opt Lett 30: 1461-1463.

36. Abramochkin EG, Volostnikov VG (2010) Phys Generalized HermiteLaguerre-Gauss Beams. Wave Phenom 18: 14-22.

37. Abramochkin E, Alieva T (2017) Closed-form expression for mutual intensity evolution of Hermite-Laguerre-Gaussian Schell-model beams. Opt Lett 42(19): 4032-4035.

38. Bandres MA, Gutierrez-Vega JC (2004) Ince-Gaussian beams. Opt Lett 29: $144-146$

39. Bandres MA, Gutierrez-Vega JC (2004) Ince-Gaussian modes of the paraxial wave equation and stable resonators. J Opt Soc Am A 21: 873880.

40. Leach J, Yao E, Padgett MJ (2004) Observation of the vortex structure of a non-integer vortex beam. New J Phys 6: 1-8.

41. Berry MV (2004) Optical vortices evolving from helicoidal integer and fractional phase steps. J Opt A Pure Appl Opt 6(2): 259-268.

42. Götte JB, O Holleran K, Preece D, Flossmann F, Franke-Arnold S, et al. (2018) Light beams with fractional orbital angular momentum and their vortex structure. Opt Express 16(2): 993-1006.

43. Agarwal GS, Banerji J (2006) Entanglement by linear SU(2) transformations: Generation and evolution of quantum vortex states. J Phys A Math Gen 39(37): 11503-11519.

44. Tuan PH, Liang HC, Huang KF, Chen YF (2018) Realizing High-PulseEnergy Large-Angular-Momentum Beams by Astigmatic Transformation of Geometric Modes in an Nd: YAG/Cr4+:YAG Laser. IEEE J Sel Top Quantum Electron 24(5).

45. Uan PHT, Sieh YHH, Ai YHL, Uang KFH, Hen YFC (2018) Characterization and generation of high-power multi-axis vortex beams by using off-axis pumped degenerate cavities with external astigmatic mode converter 26(16): 20481-20491.

46. ShenY, WanZ, Meng Y, Fu X, Gong M (2018) Polygonal Vortex Beams. IEEE Photonics J 10(4): 1503016.

47. Shen Y, Fu X, Gong M (2018) Truncated triangular diffraction lattices and orbital-angular-momentum detection of vortex SU (2) geometric modes. Opt Express 26(20): 25545-25557.

48. Beijersbergen MW, Allen L, HELO Van Der Veen, JP Woerdman (1993) Astigmatic laser-mode converters and transfer of orbital angular momentum. Opt Commun 96: 123-132.
49. Bentley JB, Davis JA, Bandres MA, Gutiérrez-vega JC (2006) Generation of helical Ince - Gaussian beams with a liquid-crystal display. 31(5): 649651.

50. Woerdemann M, Alpmann C, Esseling M, Denz C (2013) Advanced optical trapping by complex beam shaping. Laser Photonics Rev 7(6): 839-854.

51. Kulkarni G, Sahu R, Magaña-Loaiza OS, Boyd RW, Jha AK (2017) Singleshot measurement of the orbital-angular-momentum spectrum of light. Nat Commun 8(1): 1-8.

52. Errico AD, Amelio RD, Piccirillo B, Cardano F, Marrucci L (2017) Measuring the complex orbital angular momentum spectrum and spatial mode decomposition of structured light beams 4(11).

53. Alperin SN, Siemens ME (2017) Angular Momentum of Topologically Structured Darkness. Phys Rev Lett 119(20): 1-5.

54. Science $O$ (2016) Fractional vortex Hilbert's Hotel 3(3): 3-6.

55. Tkachenko G, Chen M, Dholakia K, Mazilu M (2017) Is it possible to create a perfect fractional vortex beam? Optica 4(3): 330.

56. Zhan QW (2009) Cylindrical vector beams: from mathematical concepts to applications. Advances in Optics and Photonics p. 1-57.

57. Christian Maurer, Jesacher A (2017) Tailoring of arbitrary optical vector beams. New J Phys 9: 78.

58. Vyas S, Kozawa Y, Sato S (2013) Polarization singularities in superposition of vector beams. OPTICS EXPRESS 21(7): 8972-8986.

59. Milione G, Sztul HI, Nolan DA, Alfano RR (2011) Higher-Order Poincaré Poincare' Sphere, Stokes Parameters, and the Angular Momentum of Light. PRL 107: 053601.

60. Niziev VG, Chang RS, Nesterov AV (2006) Generation of inhomogeneously polarized laser beams by use of a Sagnac interferometer. Appl Opt 45: 8393-8399.

61. Ito A, Kozawa Y, Sato S (2010) Generation of hollow scalar and vector beams using a spot-defect mirror. J Opt Soc Am A 27(9): 2072-2077.

62. Cardano F, Karimi E, Slussarenko S (2012) Polarization pattern of vector vortex beams generated by q-plates with different topological charges. APPLIED OPTICS 51(10): C1-c6.

63. Moh KJ, Yuan XC, Bu J (2007) Generating radial or azimuthal polarization by axial sampling of circularly polarized vortex beams. APPLIED OPTICS 46(30): 7544-7551.

64. Wang YC, Zhang HJ, Yu HH (2012) Light propagation in an optically active plate with topological charge. Applied Physics Letters 101: 171114.

65. Lu TH, Huang TD, Wang JG (2016) Generation of flower high-order Poincaré sphere laser beams from a spatial light modulator. Scientific Reports 6(39657): 1-6.

66. Milione G, Evans S, Nolan DA, Alfano RR (2012) Higher Order Pancharatnam-Berry Phase and the Angular Momentum of Light. PRL 108: 190401.

67. Chen SZ, Zhou XX, Liu YC (2014) Gneration of arbitrary cylindrical vector beams on the higher order Poincaré sphere. OPTICS LETTERS 39(18): 5274-5276.

68. Naidoo D, Roux FS, Dudley A, Litvin I, Piccirillo B (2016) Controlled generation of higher-order Poincaré sphere beams from a laser. Nat Photonics 10(5): 327-332.

69. Senthilkumaran P (2018) Phase engineering methods in polarization singularity lattice generation. 1(1): 193-199.

70. Otte E, Alpmann C, Denz C (2018) Polarization Singularity Explosions in Tailored Light Fields. Laser Photonics Rev 12(6): 1-11.

71. A Kovalev AL (2018) Tailoring polarization singularities in a Gaussian beam with locally linear polarization. Opt Lett 43(13): 3084-3087. 


\section{ISSN: 2574-1241}

DOI: $10.26717 / B J S T R .2018 .09 .001801$

Mali Gong. Biomed J Sci \& Tech Res

(c) (i) This work is licensed under Creative

Submission Link: https://biomedres.us/submit-manuscript.php

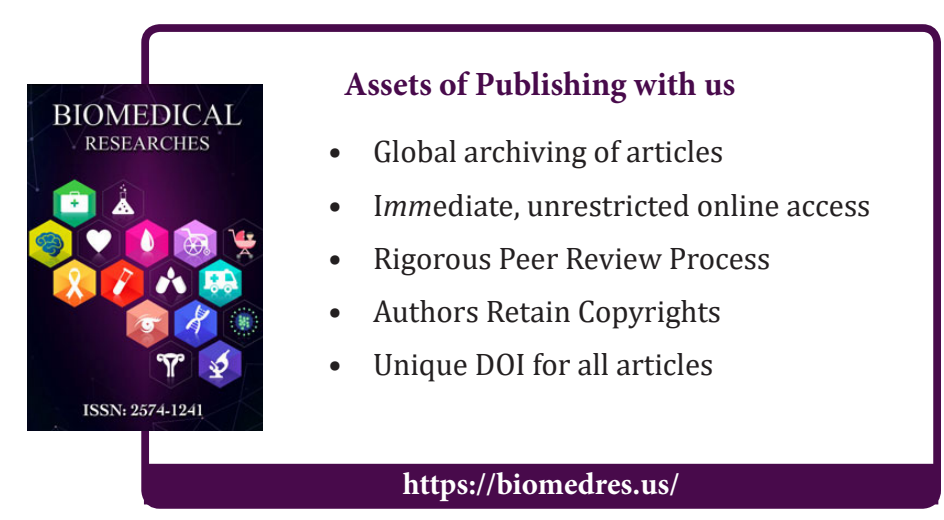

